\title{
Interactions between Kisspeptin Neurons and Hypothalamic Tuberoinfundibular Dopaminergic Neurons in Aged Female Rats
}

\author{
Kinuyo Iwata ${ }^{1}$, Masaaki Ikehara ${ }^{1}$, Yuyu Kunimura ${ }^{1}$ and Hitoshi Ozawa ${ }^{1}$ \\ ${ }^{1}$ Department of Anatomy and Neurobiology, Graduate School of Medicine, Nippon Medical School, Bunkyo-ku, Tokyo 113- \\ 8602, Japan
}

Received August 22, 2016; accepted October 24, 2016; published online December 22, 2016

\begin{abstract}
Kisspeptin neurons in the arcuate nucleus (ARC) regulate prolactin secretion, and are in physical contact with tuberoinfundibular dopaminergic (TIDA) neurons, which inhibit prolactin secretion. Prolactin levels in the blood are increased with advancing age in rats; therefore, we investigated the interactions with TIDA neurons and kisspeptin neurons in aged female rats (24 months of age), relative to those of young adult female rats (9-10 weeks of age). Plasma prolactin levels in the aged rats were significantly higher than those of young adult rats. Tyrosine hydroxylase (TH)-immunoreactive (ir) cell bodies and kisspeptin-ir nerve fibers were found in the dorsomedial ARC of both groups. The number of TH-ir cell bodies in the dorsomedial ARC did not differ significantly between groups. Additionally, no significant differences in the number of TH-ir cells in contact with kisspeptin-ir fibers was observed between groups. However, the number of kisspeptin-ir or Kiss1 mRNA-expressing cells in the ARC was significantly reduced in the aged rats compared with that of the young rats. These results suggest that the contacts between TIDA neurons and kisspeptin neurons are maintained after reproductive senescence, while production of kisspeptin in the ARC decreases significantly during aging.
\end{abstract}

Key words: kisspeptin, prolactin, tyrosine hydroxylase, aging, dopamine

\section{Introduction}

Prolactin plays an important role in reproduction, such as the promotion of lactation and reproductive behavior. In rodents, prolactin also has effects on luteal function [4]. The release of prolactin is stimulated by several factors such as thyrotropin-releasing hormone (TRH) and estrogen, and inhibited by dopaminergic neurons $[5,8]$. The dopaminergic neurons that regulate prolactin secretion are located within the hypothalamic arcuate nucleus (ARC), and are subdivided into three groups: the tuberoinfundibular (TIDA), tuberohypophyseal, and periventricular hypophyseal dopaminergic neurons [4]. The cell bodies of TIDA neurons are found in the dorsomedial ARC and project to

Correspondence to: Hitoshi Ozawa, Department of Anatomy and Neurobiology, Graduate School of Medicine, Nippon Medical School, 11-5 Sendagi, Bunkyo-ku, Tokyo 113-8602, Japan.

E-mail: hozawa@nms.ac.jp the median eminence. Therefore, dopamine secreted from TIDA neurons is released in the pituitary portal blood and inhibits prolactin release in lactotrophs through activation of $\mathrm{D}_{2}$-like receptors $[4,5]$. The activity of TIDA neurons is regulated by several neurotransmitters, neuromodulators and peripheral hormones [4]. Inhibitory agents such as serotonin, noradrenaline and estrogen are able to promote prolactin release through a decrease in TIDA activity. However, stimulatory agents such as acetylcholine, vasopressin and prolactin are able to promote the activity of TIDA neurons, resulting in decreased prolactin secretion.

Kisspeptin is a neuropeptide with an important role in the regulation of ovulation and follicular development through stimulation of $\mathrm{GnRH} /$ luteinizing hormone ( $\mathrm{LH}$ ) release in mammals [18]. In rodents, kisspeptin neurons located in the ARC coexpress neurokinin B (NKB) and dynorphin; described as KNDy neurons, these cells are thought to be involved in the generation of pulsatile GnRH/LH secretions [12]. Furthermore, kisspeptin induces 
prolactin release through inhibition of TIDA neurons in the presence of estrogen [17], and its fibers are in physical contact with TIDA neurons [19]. Kisspeptin neurons also express prolactin receptors [11] and are suppressed by prolactin [2], suggesting that kisspeptin and prolactin levels are regulated in coordination with each other.

In rats, serum prolactin levels increase with age [13, 22]. Serum prolactin levels of aged male rats (24-26 months of age) are, therefore, higher than those of young male rats (2-3 months of age) [6]. Dopamine levels in pituitary stalk blood plasma samples from aged rats are lower than those of young rats; thus, the increased incidence of hyperprolactinemia observed with advancing age may be associated with a reduction in dopamine secretion from TIDA neurons. Expression of the kisspeptin gene in the ARC of middle-aged female rats is lower than that of young rats [9]. Based upon these findings, this study aims to investigate the interaction between kisspeptin and TIDA neurons in aged female rats using immunohistochemical and in situ hybridization analysis.

\section{Materials and Methods}

\section{Animals}

Young adult ( 8 weeks of age, Tokyo Laboratory Animals Science, Tokyo, Japan) and aged (24 months of age, supplied by the Tokyo Metropolitan institute of Gerontology, Tokyo, Japan) female Wistar rats were housed in a controlled (14-hr light/10-hr dark, 6:00 lights on) environment with free access to food and water. Young rats that had undergone at least two consecutive 4-day estrus cycles were deemed suitable for investigations. Young (diestrus) and aged rats were deeply anesthetized with sodium pentobarbital, and blood samples were collected from the cervical vein. After blood sampling, anesthetized rats were perfused through the heart with a physiological saline solution, followed by $4 \%$ paraformaldehyde (PFA) in a $0.1 \mathrm{M}$ phosphate buffer $(\mathrm{PB})$. The brain was removed from the skull and stored in $4 \%$ PFA solution overnight at $4{ }^{\circ} \mathrm{C}$. The following day, the brain was immersed in $0.05 \mathrm{M} \mathrm{PB}$ containing $30 \%$ sucrose at $4^{\circ} \mathrm{C}$ for 4 days to protect against damage during freezing. One aged rat was excluded from analyses owing to the presence of a visually abnormal pituitary. Rat brains were used for immunohistochemistry and in situ hybridization analysis. All experimental procedures involving live rats were performed according to NIH guidelines (Guide for the Care and Use of Laboratory Animals) and received ethical approval from the Nippon Medical School Committee on Animal Research.

\section{Dual-labeling fluorescence immunohistochemistry with kisspeptin and tyrosine hydroxylase (TH)}

Rat brains were sectioned along the coronal plane at a thickness of $40 \mu \mathrm{m}$ using a cryostat (CM-3050-S, Leica, Wetzlar, Germany). Serial sections containing the ARC (from 1.72-4.36 mm posterior to the bregma [14]) were ob- tained. Every fourth section through the dorsomedial ARC of each animal was stained. These samples were washed in $0.1 \mathrm{M}$ PB containing $0.9 \% \mathrm{NaCl}$ and $0.3 \%$ Triton-X 100 (Wako, Osaka, Japan) (PBST), and incubated with blocking buffer (1\% bovine serum albumin (Sigma, St Louis, MO), $5 \%$ normal donkey serum and $0.02 \%$ sodium azide in PBST) for 90 min, followed by incubation with anti-rat kisspeptin antibody (1:2000, mouse monoclonal antibody, provided by Dr. Ohtaki, Takeda Pharmaceutical, Osaka, Japan) [10] and anti-TH antibody (1:5000, rabbit polyclonal antibody, Chemicon International Inc., Temecula, CA) diluted in $0.1 \mathrm{M}$ PBST at $4^{\circ} \mathrm{C}$ for 2 days. After washing in $0.1 \mathrm{M}$ PBS, the sections were incubated with Alexa Fluor 568-conjugated donkey anti-mouse IgG (1:500, Invitrogen, Eugene, OR) and Alexa Fluor 400-conjugated donkey anti-rabbit IgG (1:500, Invitrogen) diluted with $0.1 \mathrm{M}$ PBS for $2 \mathrm{hr}$ at room temperature. Sections were then washed in 0.1 M PBS, and mounted on glass slides using Vectashield (H-1400, Vector Laboratories, Burlingame, $\mathrm{CA})$ as the mounting medium. Fluorescence images were obtained using confocal laser microscopy (Fluoview FV10i, Olympus, Tokyo, Japan). Two sections with similar distributions of TH-immunoreactive (ir) cells in the dorsomedial ARC were selected from the stained sections of each animal, and Z-stack images were obtained. Z-stack images of each brain slice were acquired at $1.2-\mu \mathrm{m}$ intervals. Contacts between at least one TH-ir cell body and one kisspeptin-ir fiber were confirmed through visual inspection of images showing TH-ir cells. Numbers of TH-ir cells with, or without contact with kisspeptin fibers were counted in two sections from each animal. The total sum of the cell number was recorded ( $\mathrm{n}=4-6$ per group).

\section{In situ hybridization}

Kiss 1 in situ hybridization for Kiss1 (the gene encoding kisspeptin) was performed as described previously [1]. Briefly, serial $50-\mu \mathrm{m}$ coronal sections containing the ARC were obtained as described in the previous paragraph, and every fourth section through the ARC (from $1.72 \mathrm{~mm}$ to $4.36 \mathrm{~mm}$ posterior to the bregma) was analyzed. A Kiss 1-specific digoxigenin (DIG)-labeled probe was used to conduct free-floating in situ hybridization. A DIG-labeled antisense RNA probe was synthesized from a full-length rat Kiss1 template cDNA (GeneBank accession \#AY196983) [23] using a DIG RNA labeling kit (Roche Diagnostics, Mannheim, Germany). Every fourth section through the ARC was hybridized with the labeled probe. To visualize DIG labeling, sections were incubated with an alkaline phosphatase (AP)-conjugated anti-DIG1 antibody (1:1000, Roche Diagnostics) for $2 \mathrm{hr}$ at $37^{\circ} \mathrm{C}$ and then placed in 4-nitroblue tetrazolium chloride/5-bromo-4-chloro-3-indolyl phosphate solution (Roche Diagnostics). Kiss 1-expressing cells in the ARC were manually counted under a light microscope (BX51 microscope, Olympus, Tokyo, Japan) and the total cell number in brain sections from each animal was recorded ( $\mathrm{n}=4$ for each group). 


\section{Kisspeptin immunohistochemistry}

Serial $40-\mu \mathrm{m}$ thick sections were obtained for double staining. Every fourth section through the ARC (from 1.72 $\mathrm{mm}$ to $4.36 \mathrm{~mm}$ posterior to the bregma) was analyzed. These samples were incubated with anti-rat kisspeptin antibody (1:5000) for $24 \mathrm{hr}$ at $4^{\circ} \mathrm{C}$. Sections were washed and incubated with biotin-conjugated anti-mouse IgG (Histofine SAB-PO (M) kit, Nichirei Biosciences, Tokyo, Japan) for $2 \mathrm{hr}$, followed by incubation in avidin-biotinylated horseradish peroxidase (HRP) complex [Histofine SAB-PO (M) kit] for $2 \mathrm{hr}$. Immunoreactivity was visualized using 3'3-diaminobenzidine (DAB) mixed with $0.03 \%$ hydrogen peroxide. Kisspeptin-ir cells in the ARC were manually counted under a light microscope (BX51) and the total cell number in brain sections from each animal was recorded ( $n=4-6$ per group).

\section{Prolactin assay}

Following blood sampling, plasma was isolated immediately using centrifugation and stored at $-20^{\circ} \mathrm{C}$ until analysis using a prolactin assay. Plasma prolactin levels $(\mathrm{n}=5$ for each group) were determined using a commercial kit (rat prolactin ELISA kit, cusabio biotech, Wuhan, China). Plasma samples from aged rats were diluted fourfold with distilled water owing to readings from undiluted samples exceeding the upper limits of the detection range.

\section{Statistical analysis}

All data are reported as mean \pm standard error of the mean $(\mathrm{SEM})$. Statistical differences $(P<0.05)$ between groups were determined using an unpaired Student's $t$-test.

\section{Results}

Plasma prolactin levels in aged rats were significantly higher than those of young rats (Fig. 1). Kisspeptin-ir fibers and $\mathrm{TH}$-ir cell bodies were investigated using immunofluorescence microscopy, and were observed in the dorsomedial ARC of both aged and young rats (Fig. 2A). The numbers of $\mathrm{TH}$-ir cells did not differ significantly between groups (Fig. 2B). Furthermore, no significant differences in the number of TH-ir cells in contact with kisspeptin-ir fibers were observed (Fig. 2B). Kisspeptin-ir fibers were observed in the dorsomedial ARC of both aged and young rats, although the number of kisspeptin-ir cell bodies in the ARC was clearly lower in aged rats compared with young rats (Fig. 3). Moreover, significantly lower numbers of Kiss 1 mRNA-expressing cells were observed using in situ hybridization in the ARC of aged rats compared with those of young adult rats (Fig. 3).

\section{Discussion}

In rats, prolactin levels increase with age [6, 13, 22]. In this study, plasma prolactin levels in aged rats were also higher than those of young rats. TIDA neurons are known

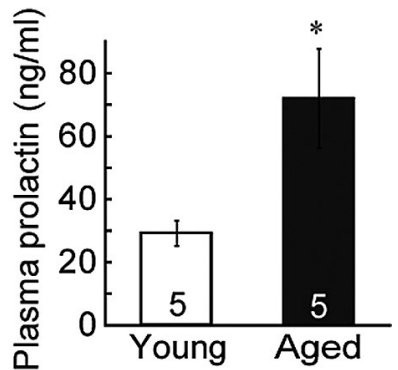

Fig. 1. Plasma prolactin concentrations in young (9-10 weeks of age) and aged (24 months of age) female rats. The statistical significance of these differences was determined using an unpaired Student's $t$-test. $* P<0.05, v s$. young rats. Young rats during the diestrus stage were used for this investigation. Values are expressed as mean \pm SEM. Numbers in each column represent the number of animals examined.

to regulate prolactin secretion [5]. Nonetheless, the number of TH-ir cells did not differ significantly between groups. This result is consistent with that of previous studies [15, 16]. No significant differences in both the number of TH-ir cells and also levels of TH mRNA expression in the hypothalamus were observed in aged, compared with young rats $[15,16]$. However, the same study demonstrated that dopamine levels in the hypophysial portal blood of aged male rats are lower than those of young male rats [15]. Also, TH activity in the medial basal hypothalamus was reduced in aged female rats compared with young female rats [15]; therefore, the high levels of prolactin in the blood of aged rats may be induced by a decrease in $\mathrm{TH}$ activity rather than a decrease in the number of TH-expressing cells.

Kisspeptin-expressing neurons in the ARC are in contact with TIDA neurons [19, 20], and regulate prolactin secretion through inhibition of TIDA neurons [17]. In this study, kisspeptin-ir fibers were found in the dorsomedial $\mathrm{ARC}$ of both young and aged rats. In addition, the number of TH-ir cells in contact with kisspeptin-ir fibers did not differ between young and aged rats. Numbers of kisspeptinir or Kiss 1 mRNA-expressing cells in the ARC were reduced in aged rats compared with young rats; therefore, kisspeptin appears to have accumulated in the terminals of fibers owing to a reduction in kisspeptin production in aged rats. It is difficult to clearly explain the mechanism of this result. It is a possible explanation that the age-related changes about the axonal transport, release mechanism and/or metabolic status of kisspeptin in the axon terminal may also be considerable. The further study on these possibilities is necessary.

Previous studies have shown that kisspeptin increases prolactin secretion through inhibition of TIDA neurons in the presence of estrogen [17]. In this study, kisspeptin expression decreased in aged rats with hyperprolactinemia. The reasons for this discrepancy are unclear. However, there is a need to take into account a short loop feedback system between kisspeptin and prolactin; kisspeptin neurons in the ARC express the prolactin receptor [11] and are 
A
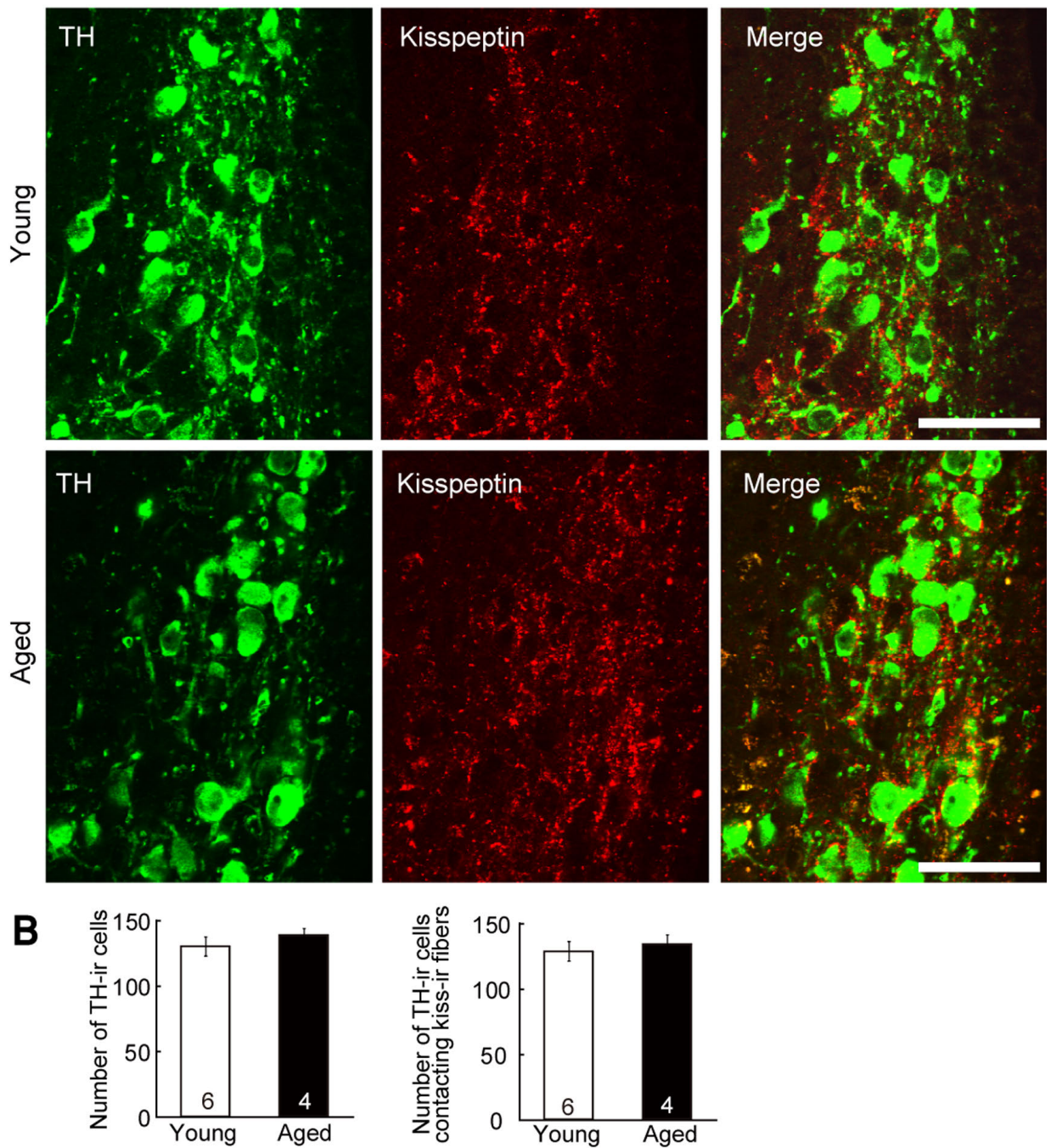

Fig. 2. Immunohistochemical labelling of tuberoinfundibular dopaminergic (TIDA) neurons and kisspeptin fibers in the dorsomedial arcuate nucleus (ARC). (A) Double immunostaining for tyrosine hydroxylase ( $\mathrm{TH}$, green) and kisspeptin (red) in the dorsomedial ARC of representative rats. Upper panels show images from the ARC of young rats during the diestrus stage. Lower panels show images from aged rats. Bars $=50 \mu \mathrm{m}$. (B) Number of THir cells (left panel) and TH-ir cells in contact with kisspeptin-ir fibers (right panel) in the dorsomedial ARC. Values are expressed as mean \pm SEM. Numbers in each column represent the number of animals examined. The statistical significance of these differences was determined using an unpaired Student's $t$-test.

inhibited by prolactin [2]. Therefore, kisspeptin may act as a trigger of hyperprolactinemia and is inhibited by the increase in serum prolactin levels in aging rats.

Some endogenous signaling molecules are able to stimulate prolactin secretion. TRH stimulates prolactin secretion from lactotrophs [8]. High levels of proTRH are expressed in the hypothalamic paraventricular nucleus (PVN). Levels of TRH mRNA expression in the hypothalamic PVN of male rats decrease with advancing age [3]; therefore, TRH levels may no longer be sufficient to stimulate prolactin secretion. Estrogen is also able to stimulate prolactin secretion, through inhibition of TIDA neurons, because TIDA neurons express the estrogen receptor $\alpha$ $(\mathrm{ER} \alpha)$, and are inhibited by estrogen [5]. In aged female rats in an anestrus state, serum estradiol levels decrease compared with those of young rats $[7,21]$. Therefore, estrogen may not be involved in prolactin secretion in aged rats.

In this study, the extent of kisspeptin production in the ARC of aged rats was reduced compared with that of young rats, although no significant differences in TH-immunoreactivity in TIDA neurons and kisspeptinimmunoreactivity in the fibers of the dorsomedial ARC were observed with aging. Therefore, contacts between kisspeptin and TIDA neurons seem to be maintained during aging. Kisspeptin neurons may affect not so much a number of TH cells as a release/metabolism of TH, which may be associated with regulation of prolactin release in aged animals. The present study of alterations in kisspeptin 

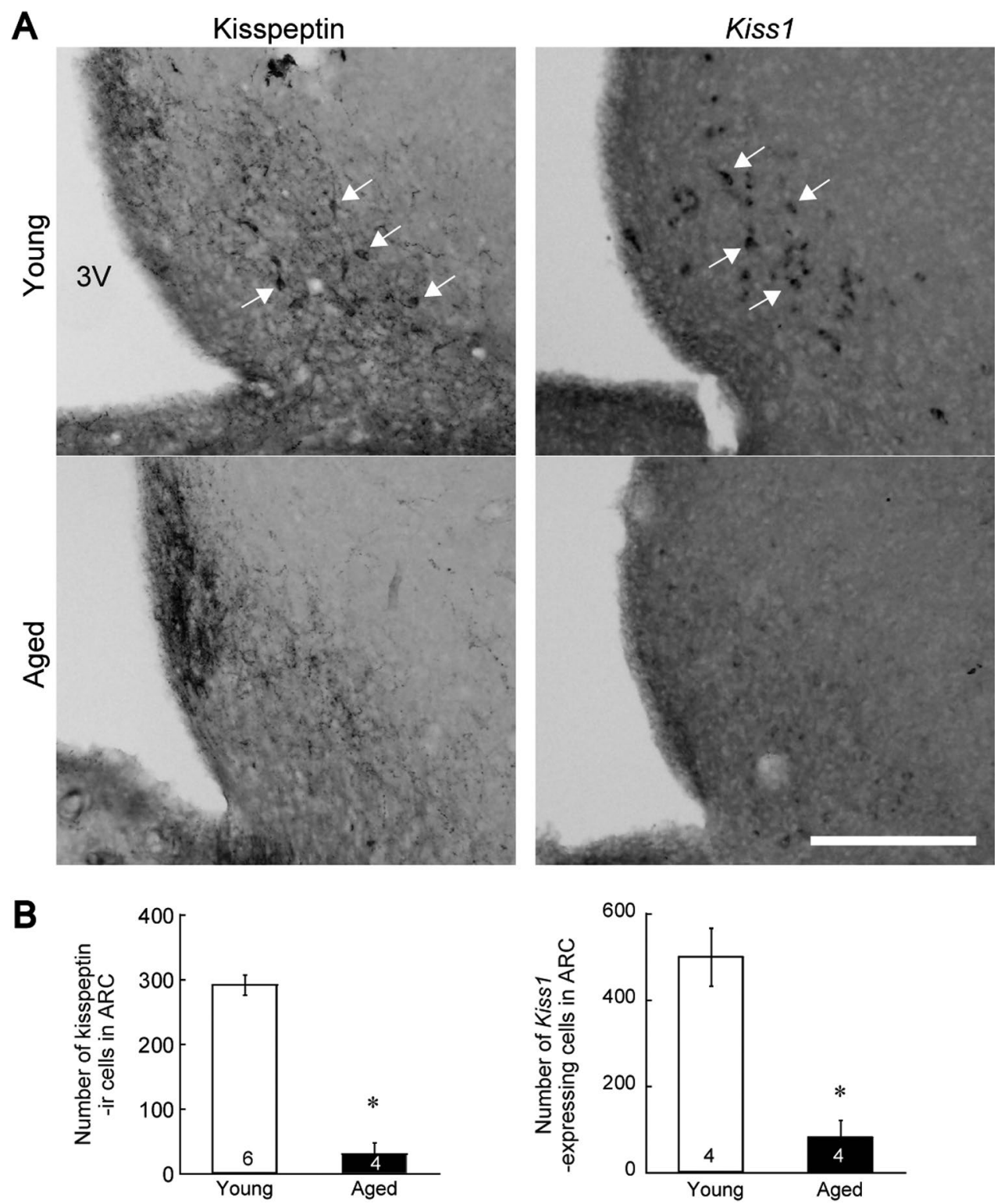

Fig. 3. Expression of kisspeptin in cell bodies in the ARC. (A) Expression of kisspeptin, as indicated by kisspeptin-immunoreactivity (left panels) or Kiss 1 mRNA, as determined using in situ hybridization (right panels) in the ARC of representative rats. Upper panels show images from the ARC of young rats during the diestrus stage. Lower panels show images from aged rats. Arrows indicate the presence of kisspeptin-immunoreactivity or Kiss 1 mRNA-expressing cell bodies. 3V, the third ventricle. Bar $=200 \mu \mathrm{m}$. (B) Number of kisspeptin-immunoreactive cells (left) and Kiss 1 mRNA-expressing cells (right) in the ARC. Values are expressed as mean \pm SEM. Numbers in each column represent the number of animals examined. The statistical significance of these differences was determined using an unpaired Student's $t$-test. ${ }^{*} P<0.05$, vs. young rats.

neurons with advancing age provides further information on the changes in reproductive function observed during aging, and during age-related hormonal alterations, such as the menopause.

\section{Disclosure Statement}

The authors have nothing to disclose.

\section{Acknowledgments}

We are grateful to Takeda Pharmaceutical Company ltd. for kindly providing rat Kiss 1 cDNA. This work was supported by Grants-in-Aid for Scientific Research (No. 26460323 to H. O. and No. $15 \mathrm{~K} 18979$ to K. I.) from the Japan Society for the Promotion of Science (JSPS) KAKENHI, and the Japanese Ministry of Education, Sports, Science. 


\section{References}

1. Adachi, S., Yamada, S., Takatsu, Y., Matsui, H., Kinoshita, M., Takase, K., Sugiura, H., Ohtaki, T., Matsumoto, H., Uenoyama, Y., Tsukamura, H., Inoue, K. and Maeda, K. (2007) Involvement of anteroventral periventricular metastin/kisspeptin neurons in estrogen positive feedback action on luteinizing hormone release in female rats. J. Reprod. Dev. 53; 367-378.

2. Araujo-Lopes, R., Crampton, J. R., Aquino, N. S., Miranda, R. M., Kokay, I. C., Reis, A. M., Franci, C. R., Grattan, D. R. and Szawka, R. E. (2014) Prolactin regulates kisspeptin neurons in the arcuate nucleus to suppress LH secretion in female rats. Endocrinology 155; 1010-1020.

3. Cizza, G., Brady, L. S., Calogero, A. E., Bagdy, G., Lynn, A. B., Kling, M. A., Blackman, M. R., Chrousos, G. P. and Gold, P. W. (1992) Central hypothyroidism is associated with advanced age in male Fischer 344/ $\mathrm{N}$ rats: in vivo and in vitro studies. Endocrinology 131; 2672-2680.

4. Freeman, M. E., Kanyicska, B., Lerant, A. and Nagy, G. (2000) Prolactin: structure, function, and regulation of secretion. Physiol. Rev. 80; 1523-1631.

5. Grattan, D. R. (2015) 60 YEARS OF NEUROENDOCRINOLOGY: The hypothalamo-prolactin axis. J. Endocrinol. 226; T101-122.

6. Gudelsky, G. A., Nansel, D. D. and Porter, J. C. (1981) Dopaminergic control of prolactin secretion in the aging male rat. Brain Res. 204; 446-450.

7. Huang, H. H., Steger, R. W., Bruni, J. F. and Meites, J. (1978) Patterns of sex steroid and gonadotropin secretion in aging female rats. Endocrinology 103; 1855-1859.

8. Joseph-Bravo, P., Jaimes-Hoy, L., Maria Uribe, R. and Charli, J. L. (2015) 60 YEARS OF NEUROENDOCRINOLOGY: TRH, the first hypophysiotropic releasing hormone isolated: control of the pituitary-thyroid axis. J. Endocrinol. 226; T85-T100.

9. Kermath, B. A., Riha, P. D., Woller, M. J., Wolfe, A. and Gore, A. C. (2014) Hypothalamic molecular changes underlying natural reproductive senescence in the female rat. Endocrinology $155 ; 3597-3609$.

10. Kinoshita, M., Tsukamura, H., Adachi, S., Matsui, H., Uenoyama, Y., Iwata, K., Yamada, S., Inoue, K., Ohtaki, T., Matsumoto, H. and Maeda, K. (2005) Involvement of central metastin in the regulation of preovulatory luteinizing hormone surge and estrous cyclicity in female rats. Endocrinology 146; $4431-4436$.

11. Kokay, I. C., Petersen, S. L. and Grattan, D. R. (2011) Identification of prolactin-sensitive GABA and kisspeptin neurons in regions of the rat hypothalamus involved in the control of fertility. Endocrinology 152; 526-535.

12. Lehman, M. N., Coolen, L. M. and Goodman, R. L. (2010)
Minireview: kisspeptin/neurokinin B/dynorphin (KNDy) cells of the arcuate nucleus: a central node in the control of gonadotropin-releasing hormone secretion. Endocrinology 151; 3479-3489.

13. Parkening, T. A., Collins, T. J. and Smith, E. R. (1980) A comparative study of prolactin levels in five species of aged female laboratory rodents. Biol. Reprod. 22; 513-518.

14. Paxinos, G. and Watson, C. (2007) The Rat Brain in Stereotaxic Coordinates, 6th ed., Academic Press, San Diego.

15. Porter, J. C., Aguila-Mansilla, N., Ramin, S. M. and Kedzierski, W. (1994) Secretion by hypothalamic dopaminergic neurons of the aged brain. Neurobiol. Aging 15; 535-539.

16. Reymond, M. J., Arita, J., Dudley, C. A., Moss, R. L. and Porter, J. C. (1984) Dopaminergic neurons in the mediobasal hypothalamus of old rats: evidence for decreased affinity of tyrosine hydroxylase for substrate and cofactor. Brain Res. 304; 215-223.

17. Ribeiro, A. B., Leite, C. M., Kalil, B., Franci, C. R., AnselmoFranci, J. A. and Szawka, R. E. (2015) Kisspeptin regulates tuberoinfundibular dopaminergic neurones and prolactin secretion in an oestradiol-dependent manner in male and female rats. J. Neuroendocrinol. 27; 88-99.

18. Roa, J., Navarro, V. M. and Tena-Sempere, M. (2011) Kisspeptins in reproductive biology: consensus knowledge and recent developments. Biol. Reprod. 85; 650-660.

19. Sawai, N., Iijima, N., Takumi, K., Matsumoto, K. and Ozawa, H. (2012) Immunofluorescent histochemical and ultrastructural studies on the innervation of kisspeptin/neurokinin B neurons to tuberoinfundibular dopaminergic neurons in the arcuate nucleus of rats. Neurosci. Res. 74; 10-16.

20. Sawai, N., Iijima, N., Ozawa, H. and Matsuzaki, T. (2014) Neurokinin B- and kisspeptin-positive fibers as well as tuberoinfundibular dopaminergic neurons directly innervate periventricular hypophyseal dopaminergic neurons in rats and mice. Neurosci. Res. 84; 10-18.

21. Steger, R. W., Huang, H. H. and Meites, J. (1979) Relation of aging to hypothalamic LHRH content and serum gonadal steroids in female rats. Proc. Soc. Exp. Biol. Med. 161; 251-254.

22. Steger, R. W. (1981) Age related changes in the control of prolactin secretion in the female rat. Neurobiol. Aging 2; 119 123.

23. Terao, Y., Kumano, S., Takatsu, Y., Hattori, M., Nishimura, A., Ohtaki, T. and Shintani, Y. (2004) Expression of KiSS-1, a metastasis suppressor gene, in trophoblast giant cells of the rat placenta. Biochim. Biophys. Acta 1678; 102-110.

This is an open access article distributed under the Creative Commons Attribution License, which permits unrestricted use, distribution, and reproduction in any medium, provided the original work is properly cited. 